\title{
Spectroscopic observations and modelling of impulsive Alfvén waves along a polar coronal jet
}

\author{
P. Jelínek ${ }^{1}$, A. K. Srivastava ${ }^{2}$, K. Murawski ${ }^{3}$, P. Kayshap ${ }^{4}$, and B. N. Dwivedi ${ }^{2}$ \\ ${ }^{1}$ University of South Bohemia, Faculty of Science, Institute of Physics and Biophysics, Branišovská 10, 37005 České Budějovice, \\ Czech Republic \\ e-mail: pjelinek@prf.jcu.cz \\ 2 Department of Physics, Indian Institute of Technology (Banaras Hindu University), 221005 Varanasi, India \\ 3 Group of Astrophysics, UMCS, ul. Radziszewskiego 10, 20-031 Lublin, Poland \\ 4 Inter University Centre for Astronomy and Astrophysics, Ganeshkhind, 411007 Pune, India
}

Received 19 May 2014 / Accepted 12 July 2015

\section{ABSTRACT}

\begin{abstract}
Context. The magnetic reconnection in the solar corona results in impulsively generated Alfvén waves, which drive a polar jet. Aims. Using the Hinode/EIS 2" spectroscopic observations, we study the intensity, velocity, and full width at half maximum (FWHM) variations of the strongest Fe XII 195.12 A line along the jet to find the signature of Alfvén waves. We numerically simulate the impulsively generated Alfvén waves within the vertical Harris current sheet, forming the jet plasma flows, and mimicking their observational signatures.

Methods. Using the FLASH code and an atmospheric model with an embedded, weakly expanding magnetic field configuration within a vertical Harris current sheet, we solve the 2.5-dimensional (2.5D) ideal magnetohydrodynamic (MHD) equations to study the evolution of Alfvén waves and vertical flows forming the plasma jet.

Results. At a height of $\sim 5 \mathrm{Mm}$ from the base of the jet, the red-shifted velocity component of Fe XII $195.12 \AA$ line attains its maximum $\left(5 \mathrm{~km} \mathrm{~s}^{-1}\right)$, which converts into a blue-shifted velocity component between the altitude of $5-10 \mathrm{Mm}$. The spectral intensity continuously increases up to $10 \mathrm{Mm}$, while the FWHM still exhibits low values with an almost constant trend. This indicates that the reconnection point within the jet's magnetic field topology lies in the corona 5-10 Mm from its footpoint anchored in the Sun's surface. Beyond this height, the FWHM shows a growing trend. This may be the signature of Alfvén waves that impulsively evolve, due to reconnection, and propagate along the jet. From our numerical data, we evaluate space- and time- averaged Alfvén waves velocity amplitudes at different heights in the jet's current sheet, which contribute to the non-thermal motions and spectral line broadening. The synthetic width of Fe XII 195.12 A line exhibits a similar trend of increment as in the observational data, possibly proving the existence of Alfvén waves, impulsively generated by reconnection, that propagate along the jet.

Conclusions. The numerical simulations show that the impulsive perturbations in the transversal component of velocity at the reconnection point can excite the Alfvén waves. These waves can power the plasma jet higher into the polar coronal hole, as vertical plasma flows are also associated with these waves due to pondermotive force. The simulated Alfvén waves match well with the observed non-thermal broadening along the jet, which may provide direct spectroscopic evidence of the impulsively excited Alfvén waves within the polar jet.
\end{abstract}

Key words. magnetohydrodynamics (MHD) - Sun: atmosphere - Sun: corona - waves - methods: numerical

\section{Introduction}

Polar coronal jets are well-observed, large-scale, and confined plasma transients in the solar atmosphere (see Nisticò et al. 2009; Nisticò \& Zimbardo 2012). They can significantly contribute to the energy transport and the formation of nascent supersonic wind. The exact driving mechanisms of these kinds of jets are still debated. Broadly speaking, the two candidates, namely magnetic reconnection and magnetohydrodynamic (MHD) waves, are known to trigger these jets (e.g. Shibata 1982; Yokoyama \& Shibata 1995; Cirtain et al. 2007; Nishizuka et al. 2008; Filippov et al. 2009; Pariat et al. 2009, 2015; Srivastava \& Murawski 2011; Kayshap et al. 2013a,b, and references cited therein). The observations of Alfvén waves associated with the polar coronal jets are either related to the imaging observations of photospherically driven waves propagating along the jet (Cirtain et al. 2007) or some transversal perturbations evolved during the reconnection at the base of the jet
(Nishizuka et al. 2008). These Alfvén waves are also ubiquitous in the localized magnetic structures (e.g. spicules, prominences, small-scale chromospheric flux tubes) as well as in the largescale corona (e.g. De Pontieu et al. 2007; Okamoto et al. 2007; Tomczyk et al. 2007; Jess et al. 2009; Mathioudakis et al. 2013, and references cited therein).

Detection of Alfvén wave in solar magnetic structures is not yet well established, as these waves are incompressible. In the polar coronal plasma, these waves are observed in the form of spectral line profile variations (Banerjee et al. 1998; Harrison et al. 2002). Narrowing of the spectral line-width is attributed to the dissipation of small-amplitude Alfvén waves (Harrison et al. 2002; O'Shea et al. 2005; Bempord \& Abbo 2012; Dwivedi et al. 2014, and references cited therein). The growth and dissipation of Alfvén waves have been modelled in the corona, which also explain the observed line-width variations (e.g. Pekenulu et al. 2002; Dwivedi \& Srivastava 2006; Chmielewski et al. 2013, 2014). In particular, Chmielewski et al. (2013) reported that the 
observed line broadening, particularly in the polar corona, can be explained in terms of impulsively generated non-linear Alfvén waves. Non-linear Alfvén waves may be the likely candidates for transporting energy in the solar corona (Murawski et al. 2015a). These waves can also power the large-scale, as well as confined plasma transients, e.g. solar jets, nascent wind, and plasma flows (Murawski et al. 2015b).

It has been reported that the Alfvén waves power various jets (e.g. X-ray jets, spicules) and exist in a variety of coronal magnetic structures, however, they are identified as kink waves (Van Doorsselaere et al. 2008a,b). The existence of mixed radial and azimuthal waves are also reported in theory and observations (Goossens et al. 2009, 2012; Tian et al. 2012; Srivastava \& Goossens 2013, and references cited therein). Numerical simulations support the general interpretation of the observed oscillations as a coupling of the kink and Alfvén waves (see e.g. Pascoe et al. 2010, 2011). Kamio et al. (2010) reported the spectroscopic observations of a rotating coronal jet and interpreted it as an evolution of kink waves and instability. The solar jets are found to be driven by various physical processes, e.g. the direct magnetic reconnection generated Lorentz force (Nishizuka et al. 2008), the reconnection generated pulse (Srivastava \& Murawski 2011), the emergence and internal reconnection in small-scale kinked fluxtubes (Kayshap et al. 2013a,b), etc. However, the pure Alfvén waves driven coronal jets are difficult to detect because of observational constraints and the dynamic nature of the jet's typical magnetic field and plasma configuration.

In the present paper, we study the Hinode/EIS spectroscopic observations of a polar jet. We find the spectroscopic signatures of impulsively generated Alfvén waves and associated plasma flows within the jet. We model the observed physical processes (waves and flows) in the jet as a natural consequence of reconnection generated velocity pulse in the vertical and gravitationally stratified Harris current sheet lying in the appropriate model atmosphere with VAL-III C temperature (Vernazza et al. 1981). The structure of the present paper is as follows. In Sect. 2, we present the observational results. Section 3 describes numerical model, governing equations, initial conditions, perturbations, and numerical solutions. In Sect. 4, the numerical results are shown. The discussion and conclusions are outlined in the last section.

\section{Hinode/EIS observations of a polar coronal jet}

\subsection{Spectroscopic data and observational analyses}

A polar jet is observed using $2^{\prime \prime}$ slit scan by EUV Imaging Spectrometer (Culhane et al. 2006) onboard Hinode on 22 April, 2009. It is an established fact that the 40" and 266" slots observe the temporal image data, while $1^{\prime \prime}$ and $2^{\prime \prime}$ slits are appropriate for the spectroscopic observations of the solar corona and transition region (TR). The EIS observes high-resolution spectra in two wavelength intervals, i.e. $170-211$ and 246-292 A, using its short-wavelength (SW) and long-wavelength (LW) CCDs, respectively. The observed data contain spectral line pro-

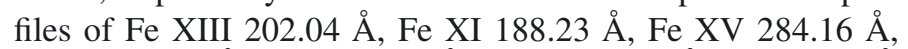
Fe XII 195.12 A, Fe X 184.54 ̊, Si VII 275.35 A, O V $192.9 \AA$ and He II $256.32 \AA$. The scanning started at 05:31:43 UT and ended at 06:33:29 UT. The exposure time on each scanning step was $31 \mathrm{~s}$. The polar jet and related plasma column, which were moving off the limb, are scanned fully in a single spatio-temporal step at 06:29 UT because they went straight into the corona. This provides us with an opportunity to understand the wave activity along the jet, which already reached up to a certain height in the polar corona. The $2^{\prime \prime}$ slit started scanning steps over the polar coronal hole with $(X$ cen, $Y$ cen $) \sim\left(30^{\prime \prime}, 939^{\prime \prime}\right)$. The observation window on the CCDs is 400 pixels high along the slit with a width of 40 pixels in the horizontal direction. The $Y$-direction covers the solar atmosphere from 740" to $1139^{\prime \prime}$ (400 pixels with $1^{\prime \prime} /$ pxiel). The direction of dispersion has the spectral resolution of 0.02 .

We apply standard EIS data reduction procedures and calibration files to the data acquired at the EUV-telescope, which is the raw (zeroth-level) data. The subroutines can be found in the sswidl software tree ${ }^{1}$. These standard subroutines correct for dark-current subtraction, cosmic-ray removal, flat-field correction, hot pixels, warm pixels, and bad or missing pixels. The data are saved in the level-1 data file, while associated errors are saved in the error file. We choose the strong line Fe XII $195.12 \AA$ to examine the spatial variations of the intensity, Doppler velocity, and full width at half maximum along the jet from its base get the clues of transversal waves. We co-align the Fe XII $195.12 \AA$ map w.r.t. the long-wavelength CCD observations of He II $256.32 \AA$ by considering it as a reference image and by estimating the offset. The orbital and slit-tilt are also corrected for data using the standard method described in the EIS software notes. We perform the double Gaussian fitting for the removal of the weak blend of Fe XII $195.18 \AA$ line that affects the line profile of Fe XII 195.12 $\AA$. The fitting function is a Gaussian for the line profile (see gauss_.pro in SolarSoft), and a straight line for background continuum (see line_.pro in SolarSoft). The fitting of the observed spectral line profile gives peak intensity, centroid (measure of flows), and Gaussian width (measure of thermal and non-thermal motions). We apply the procedure described by Young et al. (2009) in this context, which is also available in the EIS Software Note 17. We constrain Fe XII 195.18 Alline to have the same width as the Fe XII $195.12 \AA$ line. We search for the blend line Fe XII 195.18 $\AA$ within the range of $+0.06 \AA$ w.r.t. the centroid of the main line Fe XII $195.12 \AA$. We assume and require that the contribution of the blended line is searched within maximum $28 \%$ limit compared to the peak intensity of the main Fe XII $195.12 \AA$ line. We perform the fitting over binned data of 2 pixel $\times 6$ pixel to increase the signal-to-noise ratio and to obtain the reasonable fitting and estimated parameters (cf. Figs. 1 and 2). The example of the Fe XII $195.12 \AA$ fitted spectra at 14 points along the chosen slit along jet are shown in Fig. 2. These fitted profiles of Fe XII $195.12 \AA$ are free from any contribution of the weak blend of Fe XII 195.18 ̊. The 14 spatial points along the jet correspond to various heights for which the spectral parameters are derived (Fig. 3).

The wavelength calibration and estimation of the reference wavelength of Fe XII 195.12 A line is performed using the limb method (Peter \& Judge 1999). The box is chosen at the north pole limb from where the integrated spectra is derived (cf. Fig. 1, intensity map). It is likely that in and outwards motions near the limb almost cancel each other out. Therefore, the centroid of the line profile represents the rest wavelength of particular emission line. The Gaussian fitting gives the estimate of the reference wavelength as $195.128 \AA$. This reference wavelength is used in deriving the Doppler velocities in the region of our interest (cf. Figs. 1 and 3).

\footnotetext{
1 http://www.darts.isas.jaxa.jp/pub/solar/ssw/hinode/ eis/
} 

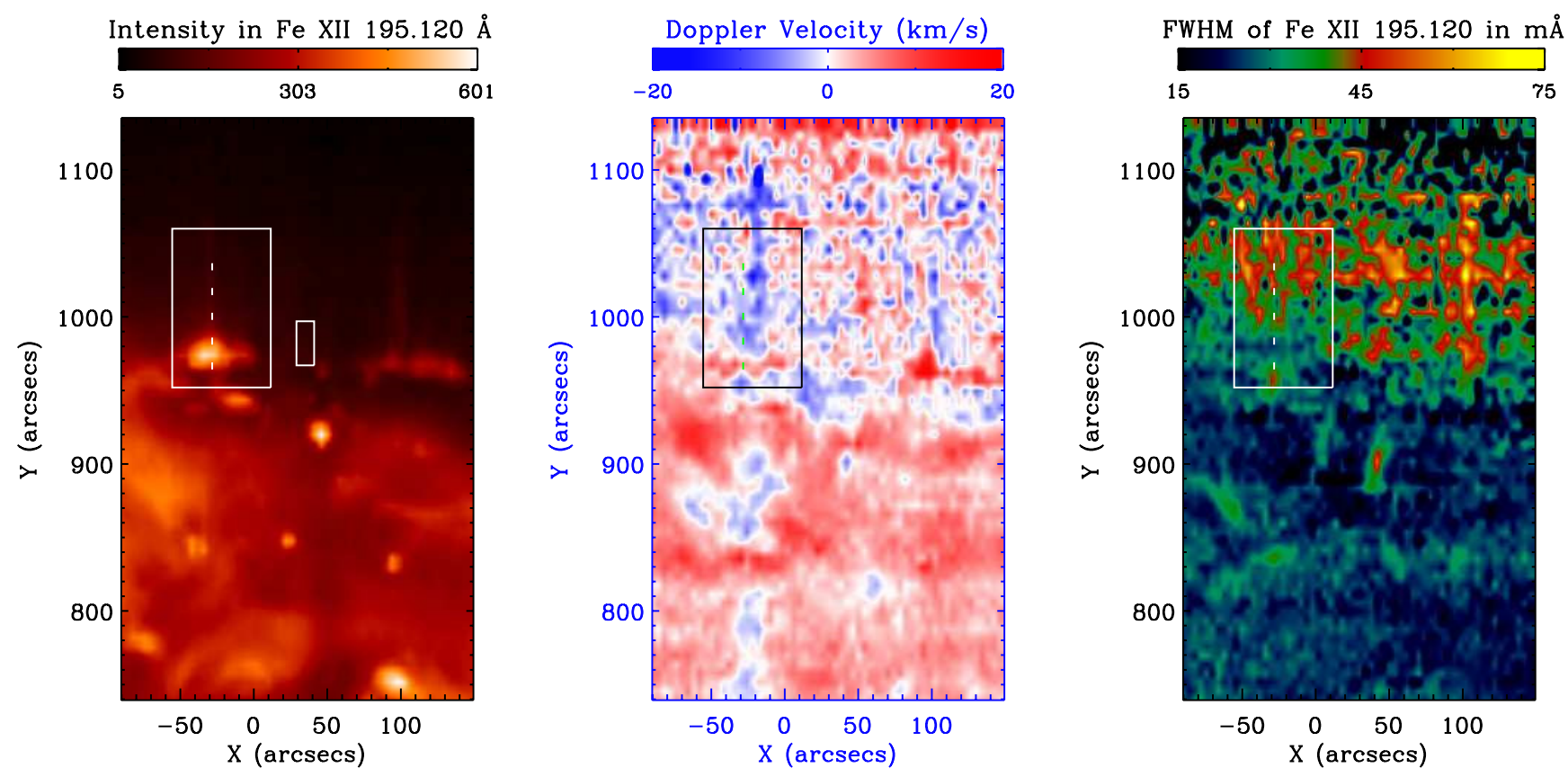

Fig. 1. Intensity, Doppler velocity, and FWHM maps of Fe XII $195.12 \AA$ line, showing the polar jet moving off the limb outwards. The white line is the position of the slit along the jet upon which the spectral parameters are estimated. The footpoint of the jet where the slit's lowest part lies, has the coordinates of $\left(-28^{\prime \prime}, 964^{\prime \prime}\right)$. The white rectangular box is chosen at the limb of the north polar corona from where the integrated line profile of Fe XII $195.12 \AA$ is derived and fitted to obtain its rest wavelength.

\subsection{Observational results}

Figure 1 displays the intensity (left), Doppler-velocity (middle), FWHM (right) maps of the polar coronal hole where the straight coronal jet has already moved off the limb. The maps are related with spectral measurements over 2 pixel $\times 6$ pixel binned data with good signal-to-noise ratio. The jet is visible in the left-most part of the intensity map, and it has a typical inverted Y-shape structure (cf. within the box in the intensity map). At the same place, in the Doppler velocity map we see the red-shifted base of the jet while the plasma moves outwards (blue shift) in the higher plasma column of the jet (cf. within the box in the Doppler velocity map). The blue shift and red shift measured in the off-limb jet correspond to the line-of-sight component of velocity variations. It seems that the jet is slightly tilted towards us. Therefore, at a certain height, the jet plasma that is outflowing towards us provides a blue shift signature, while the downflowing plasma towards the Sun within the jet gives the red shift. In the FWHM map, we find the increased contribution of the line width in the jet plasma column (cf. within the box in the line-width map). Also, the jet is not visible in Si VII 275.35 line $\left(\log T_{f} \approx 5.0\right)$, thereby confirming that it is not formed by cool transition region plasma. The jet is also not evident at higher temperatures. It is found that mostly its plasma is maintained at the inner coronal temperature of 1.0 MK. Therefore, the jet is best observed in Fe XII 195.12 A line. We do not conjecture that it is made up of a single temperature plasma. In the given observational baseline, its plasma temperature can on average be around the formation temperature of Fe XII line, i.e. $\log T_{f} \approx 6.1$.

We choose a straight slit from the base of the jet in the outwards direction along the jet plasma column (cf. Fig. 1). We measure the variation of intensity (left panel), Doppler velocity (middle panel), and FWHM (right panel) of Fe XII $195.12 \AA$ line along this chosen path (cf. Fig. 3). The first position at the bottom of the slit is the base of the jet, which is referenced as "zero Mm". The rest of the slit represents the progressive height along the jet scaled in $\mathrm{Mm}$. The total height of the slit along the jet is $56 \mathrm{Mm}$ (cf. Fig. 3). We consider the estimations of these spectral parameters along the jet up to only those heights $(\approx 55 \mathrm{Mm})$ where spectral fitting is reasonable with fewer fitting errors (cf. Fig. 2). It is clear from these panels that intensity is continuously enhanced up to $10 \mathrm{Mm}$, and decreases thereafter Doppler velocity changes from its maximum value of red shift about $+5 \mathrm{~km} \mathrm{~s}^{-1}$ from the base of the jet to the blue shift (outflows) at a height between 5-10 Mm. We find the variation of FWHM along the same path. It is almost constant without much variation up to $10-15 \mathrm{Mm}$ (cf. Fig. 3, right panel). Above this height, the FWHM increases from $30 \mathrm{~m} \AA$ to $55 \AA$ with a significant positive gradient (cf. red-fitted line in the right panel of Fig. 3). The red line shows second order polynomial fit on the FWHM data.

\subsection{Physical interpretations of the observational results}

The small-scale and low-lying bright-point loops form the wide base of the jet. They reconnect with pre-existing open field lines and propel the jets' plasma. This scenario is in accordance with the model given by Yokoyama \& Shibata (1995) about the coronal jet formation. The red shift is maximum $\left(+5 \mathrm{~km} \mathrm{~s}^{-1}\right)$ at $5 \mathrm{Mm}$ height, which means the plasma is downflowing at this location. Above this height between 5-10 Mm, this red shift is transformed into blue shift. This vertical region acts as a reconnection region where plasma flows in both directions: the downflows in small-scale loops forming the base of the jet and upflows along open field lines of the jet forming its plasma motion higher into the corona. The region where downflows (red shift) convert into outflows (blue shift) and intensity (thus density) tends towards its maximization indicates that reconnection already drove the energy release below this location near the base of the jet. The reconnection propels the plasma outwards along the open field lines of the jet. The maximum observed blue shift is $7.5 \mathrm{~km} \mathrm{~s}^{-1}$ along the jet, however, the jet is projected 

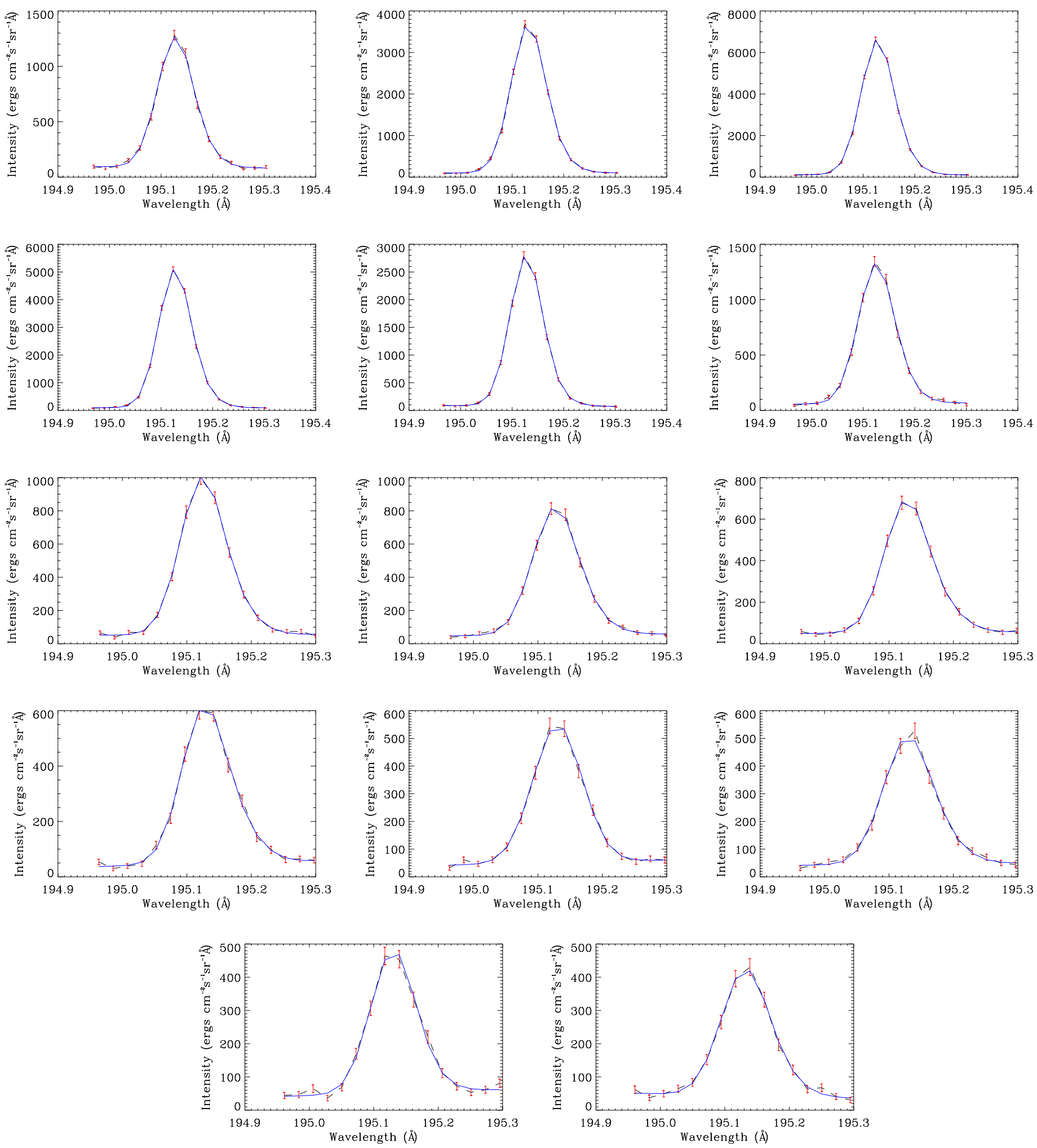

Fig. 2. Gaussian fit to the spectra derived on the chosen path along the jet. The white slit on the jet (cf. Fig. 1) is divided into 14 super pixels, each of the size of $2 \times 6$ pixel square. The line profile is derived from each super pixel (dotted blue), and the corresponding estimation of plasma parameters are made by fitting the double Gaussian (solid blue).

off the limb at the north pole. The plasma motions along the jet may have minimum contribution to the line-of-sight (LOS) Doppler shift. Therefore, the estimated Doppler velocity should be considered an apparent lower-bound velocity, which is not the actual speed of the jet. This simply provides the signature of the outflowing plasma along the jet. The FWHM also shows the increasing trend beyond 10-15 Mm height. The FWHM value exactly at the base of the jet at the north polar limb is somewhat higher, i.e. $40 \mathrm{~m} \AA$. We can discard it because of the limb effects. The contribution of stray light from continuum near the limb 

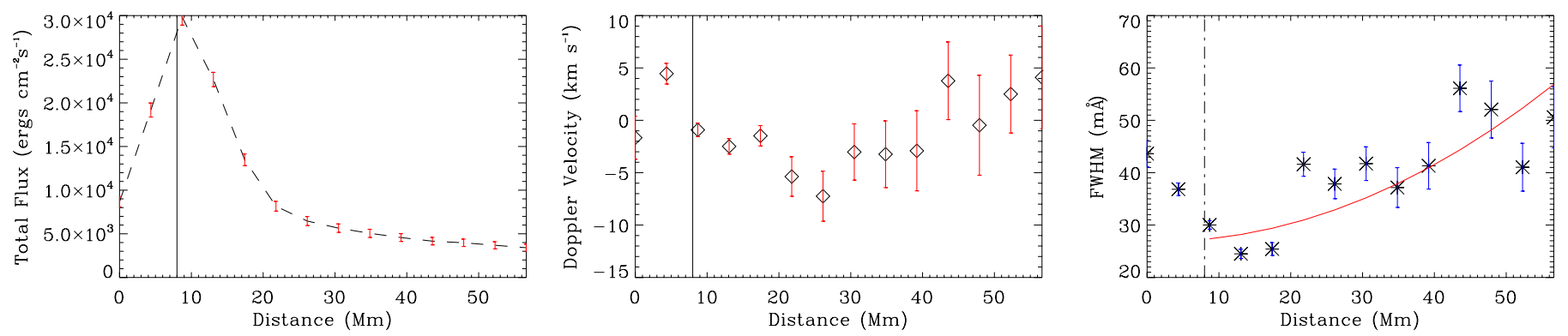

Fig. 3. Variation of intensity, Doppler velocity, and FWHM of Fe XII $195.12 \AA$ line along the path chosen on the polar jet from its base outwards. The red line shows second order polynomial fit to the FWHM data.

may affect the line profile and its width. The second point of estimated FWHM lies even higher around $36 \mathrm{~mA}$ at $5 \mathrm{Mm}$ where the highest red shift (downflow) is observed. At 5.0 Mm height, the energy release is likely to be present as downflow is maximum at this location. The energy release may cause the linewidth broadening of the spectral line (Somov 1994). Therefore, the spatial points that lie in the lower segment of the jet closer to the energy release site in the spatial range 5-15 Mm from Jet's footpoint, possess comparatively higher values of FWHM. As we move away from the energy release site at higher heights, the jet's temperature is uniform near the formation temperature of optically thin Fe XII $195.12 \AA$ line. Therefore, the contribution to the FWHM increment may be mainly due to the increment in non-thermal motions.

The reconnection generates the outflowing plasma along jet's open magnetic field lines, as well as the downflowing plasma along the small-scale loops forming its base. This region $(0-15 \mathrm{Mm})$, therefore, may be the most likely place in the lower segment of the jet around which the reconnection and related physical processes occurred. Beyond this region, the FWHM of the observed spectral line increases significantly because of non-thermal motions, as evident in the observational baseline. This FWHM broadening may be associated with the evolution and growth of the non-linear and impulsively generated Alfvén waves propagating along the open field lines of the jet away from the reconnection site higher into the corona. In theory as well as observations, Alfvén waves are found to be excited in the largescale polar coronal holes (Banerjee et al. 1998; Chmielewski et al. 2013).

The FWHM also shows varying trends in and around the observed jet, however, these trends are not so systematic and closely correlated with intensity and Doppler velocity variations with height as we observed within the jet. We do not present these plots. However, we have examined the region outside the jet's plasma column and its inverted Y-shape base in its western side. The intensity decreases rapidly as we move outside the limb, which is an obvious trend. However, there is no trend in the Doppler velocity. Doppler velocity only shows weak downflows. The FWHM also does not show any trend in its variation with height. In conclusion, present observations demonstrate the signature of impulsive Alfvén waves along the jet above the energy release site near its footpoint. In the next section, we describe our model of impulsive Alfvén waves in the 2D vertical current sheet to match with some observed properties of these wave modes.

\section{Numerical model of impulsive Alfvén waves in 2D vertical Harris current sheet}

Keeping in mind the impulsive generation of Alfvén waves due to energy release within the observed jet, we model the propagation of a transverse pulse in a $2 \mathrm{D}$ vertical Harris current sheet. Above the reconnection point, perturbations may evolve in the form of Alfvén waves propagating at various higher heights as well as the driven coupled plasma flows. We consider the height of $5 \mathrm{Mm}$ in our model to launch the reconnection generated velocity pulse in a $2 \mathrm{D}$ vertical current sheet.

\subsection{Governing equations}

In our numerical model of a Harris current sheet, embedded in a gravitationally stratified solar atmosphere, the plasma dynamics is described by the following 2.5-dimensional (2.5D) timedependent ideal MHD equations (cf. Priest 1982; Chung 2002):

$$
\begin{aligned}
& \frac{\mathrm{D} \varrho}{\mathrm{D} t}=-\varrho \nabla \cdot \boldsymbol{v}, \\
& \varrho \frac{\mathrm{D} \boldsymbol{v}}{\mathrm{D} t}=-\nabla p+\boldsymbol{j} \times \boldsymbol{B}+\varrho \boldsymbol{g}, \\
& \frac{\mathrm{D} \boldsymbol{B}}{\mathrm{D} t}=(\boldsymbol{B} \cdot \nabla) \boldsymbol{v}, \\
& \frac{\mathrm{D} e}{\mathrm{D} t}=-\gamma e \nabla \cdot \boldsymbol{v}, \\
& \nabla \cdot \boldsymbol{B}=0 .
\end{aligned}
$$

Here $\mathrm{D} / \mathrm{D} t \equiv \partial / \partial t+\boldsymbol{v} \cdot \nabla$ is the total time derivative, $\varrho$ is the mass density, $\boldsymbol{v}$ is the flow velocity, $\boldsymbol{B}$ is the magnetic field, and $\boldsymbol{g}=\left[0,-g_{\odot}, 0\right]$ is the gravitational acceleration, which is oriented in the negative $y$-direction, with $g_{\odot}=274 \mathrm{~m} \mathrm{~s}^{-2}$. Our model does not include the radiative and thermal conductive losses. This model may not fully describe the reconnection generated heating and related additional plasma evolution within the jet as we do not invoke the non-classical thermodynamical terms (e.g. radiative and thermal losses) in the governing energy equation. We only aim to understand the non-linear wave dynamics and associated vertical flows along the jet.

The current density $\boldsymbol{j}$ in Eq. (2) is expressed as

$\boldsymbol{j}=\frac{1}{\mu_{0}}(\nabla \times \boldsymbol{B})$

where $\mu_{0}=1.26 \times 10^{-6} \mathrm{H} \mathrm{m}^{-1}$ is the magnetic permeability of free space.

The internal energy density, $e$, in Eq. (4) is given by

$e=\frac{p}{\gamma-1}$

with the adiabatic coefficient, which we set and hold fixed as $\gamma=5 / 3$. 


\subsection{Gravitationally stratified Harris current sheet}

For a static $(\boldsymbol{v}=\mathbf{0})$ equilibrium, the Lorentz and gravity forces must be balanced by the pressure gradient in the entire physical domain

$-\nabla p+\boldsymbol{j} \times \boldsymbol{B}+\varrho \boldsymbol{g}=\mathbf{0}$.

The solenoidal condition, $\nabla \cdot \boldsymbol{B}=0$, is identically satisfied by the magnetic flux function, $\boldsymbol{A}$,

$\boldsymbol{B}=\nabla \times \boldsymbol{A}$.

For calculating the magnetic field in the vertically oriented Harris current sheet, we use the magnetic flux function $\boldsymbol{A}=$ $\left[0,0, A_{z}\right]$ as (Galsgaard \& Roussev 2002; Jelínek et al. 2012)

$A_{z}=-B_{0} w_{\mathrm{cs}} \ln \left\{\left|\cosh \left(\frac{x}{w_{\mathrm{cs}}}\right)\right|\right\} \exp \left(-\frac{y}{\lambda}\right)$.

Here the coefficient $\lambda$ denotes the magnetic scale height. The symbol $B_{0}$ is used for the external magnetic field and $w_{\mathrm{cs}}$ is the half-width of the current sheet. We set and hold fixed $w_{\mathrm{cs}}=$ 1.0 Mm. Substituting Eq. (10) into Eq. (9) we obtain the equations for the magnetic field in the $x-y$ plane as (Galsgaard \& Roussev 2002; Jelínek et al. 2012)

$$
\begin{aligned}
& B_{x}(x, y)=B_{0} \frac{w_{\mathrm{cs}}}{\lambda} \ln \left[\cosh \left(\frac{x}{w_{\mathrm{cs}}}\right)\right] \exp \left(-\frac{y}{\lambda}\right), \\
& B_{y}(x, y)=B_{0} \tanh \left(\frac{x}{w_{\mathrm{cs}}}\right) \exp \left(-\frac{y}{\lambda}\right) .
\end{aligned}
$$

Equation (8), while written in terms of its components, attains the following form:

$$
\begin{aligned}
& \frac{\partial p(x, y)}{\partial x}+j_{z} B_{y}(x, y)=0, \\
& \frac{\partial p(x, y)}{\partial y}-j_{z} B_{x}(x, y)+\varrho g(x, y)=0 .
\end{aligned}
$$

Here $j_{z}$ is the only non-zero component of the electric current density $\boldsymbol{j}$, see Eq. (6), given by $j_{z}=\frac{1}{\mu_{0}}(\nabla \times \boldsymbol{B})_{z}$. The condition of integrability of the above equations leads to

$\mu_{0} g \frac{\partial \varrho(x, y)}{\partial x}=\nabla \cdot\left(\mu_{0} j_{z} \boldsymbol{B}\right)$

from which we can derive the formulae for the distribution of the mass density (cf. Galsgaard \& Roussev 2002; Jelínek et al. 2012)

$$
\begin{aligned}
\varrho(x, y)= & \left\{\frac{B_{0}^{2}}{\mu_{0} g \lambda}\left\{1+\ln \left[\cosh \left(\frac{x}{w_{\mathrm{cs}}}\right)\right]\right\} \operatorname{sech}^{2}\left(\frac{x}{w_{\mathrm{cs}}}\right)\right. \\
& \left.+\varrho_{0}\right\} \cdot \exp \left(-2 \frac{y}{\lambda}\right)
\end{aligned}
$$

and the gas pressure,

$$
\begin{aligned}
p(x, y)= & \left\{\frac{B_{0}^{2}}{2 \mu_{0}} \operatorname{sech}^{2}\left(\frac{x}{w_{\mathrm{cs}}}\right)+\frac{B_{0}^{2} w_{\mathrm{cs}}^{2}}{2 \mu_{0} \lambda^{2}} \ln ^{2}\left[\cosh \left(\frac{x}{w_{\mathrm{cs}}}\right)\right]\right. \\
& \left.+\frac{\varrho_{0} g \lambda}{2}\right\} \cdot \exp \left(-2 \frac{y}{\lambda}\right)+p_{0} .
\end{aligned}
$$

Here $\varrho_{0}$ and $p_{0}$ are integration constants. The corresponding plasma temperature is assumed to be $T=1.25 \times 10^{6} \mathrm{~K}$.

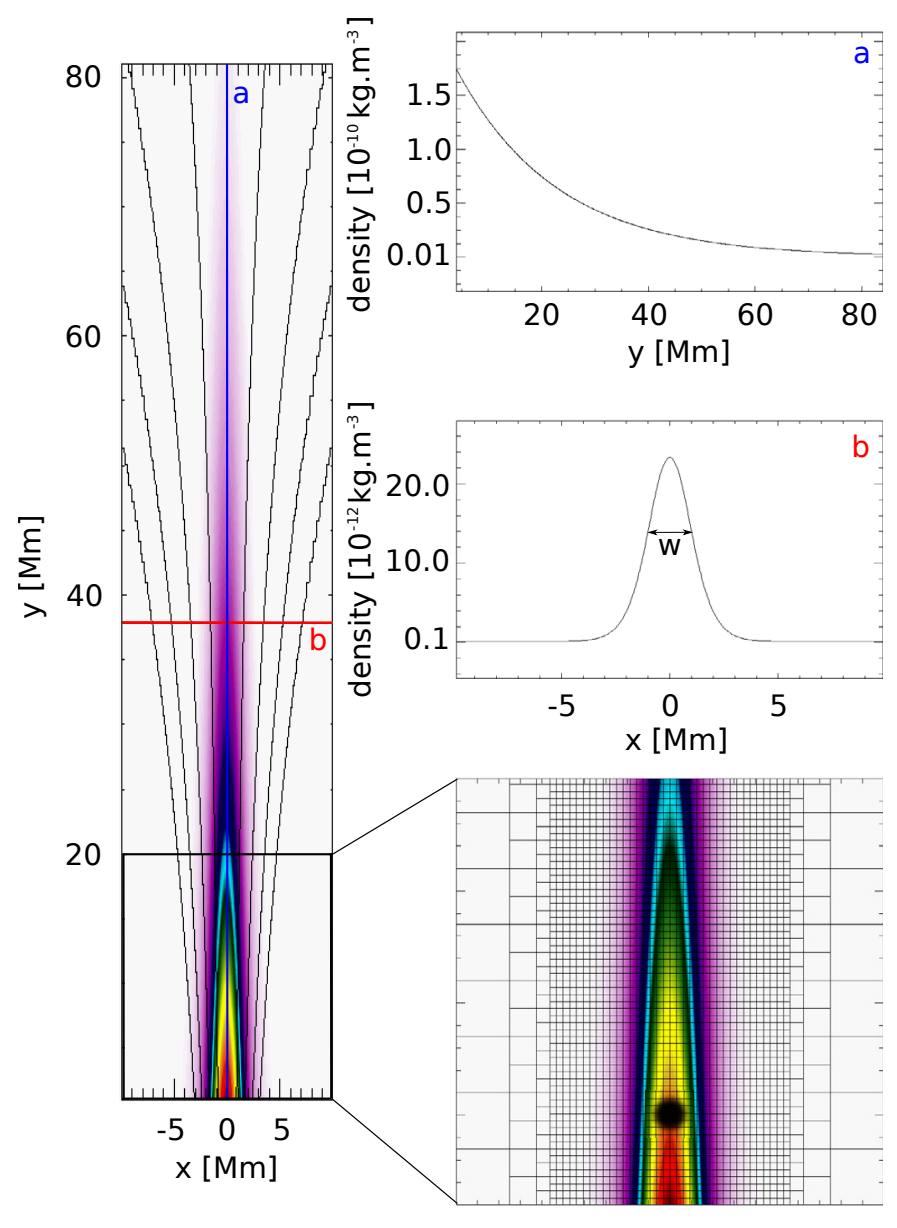

Fig. 4. Mass density distribution in the Harris current sheet, where the black contours represent the structure of magnetic field. On the righthand side of the figure both a) vertical (for $x=0 \mathrm{Mm}$ ) and b) horizontal (for $y=37.65 \mathrm{Mm}$, which is magnetic scale height) slices in mass density are shown, as well as the details of the part of the simulation region illustrating the computational grid, with adaptive mesh refinement (AMR). At the altitude $y=5 \mathrm{Mm}$ the perturbation point, $L_{\mathrm{P}}$, is marked by full black circle.

\subsection{Initial perturbation}

At the start of the numerical simulation $(t=0 \mathrm{~s})$, the equilibrium is perturbed by the Gaussian pulse in the $z$-component of velocity and has the following form (e.g. Nakariakov et al. 2004, 2005):

$V_{z}(x, y, t=0)=-A_{0} \cdot \frac{x}{\lambda} \cdot \exp \left[-\frac{x^{2}+\left(y-L_{\mathrm{P}}\right)^{2}}{\lambda^{2}}\right]$,

where $A_{0}$ is the amplitude of the initial pulse and $\lambda=4 \mathrm{Mm}$ is its width. This pulse preferentially triggers Alfvén waves. The perturbation point, $L_{\mathrm{P}}$, is located on the axis of the Harris current sheet, at a distance of $5 \mathrm{Mm}$ from the bottom boundary of the simulation region (see full black circle in Fig. 4). We set the detection points, $L_{\mathrm{D}}$, on the current-sheet axis, at the distance between the perturbation and detection points $\Delta \equiv\left|L_{\mathrm{D}}-L_{\mathrm{P}}\right|=$ $10,15,20,25,30,35,40,45,50$, and $55 \mathrm{Mm}$.

In the $2.5 \mathrm{D}$ model, the Alfvén waves decouple from magnetoacoustic waves. They can be described solely by $V_{z}(x, y, t)$. As a result, the initial pulse of Eq. (18) triggers Alfvén waves, which are approximately described in the linear case by the wave equation

$\frac{\partial^{2} V_{z}(x, y, t)}{\partial t^{2}}=c_{\mathrm{A}}^{2}(x, y) \frac{\partial^{2} V_{z}(x, y, t)}{\partial s^{2}}$, 

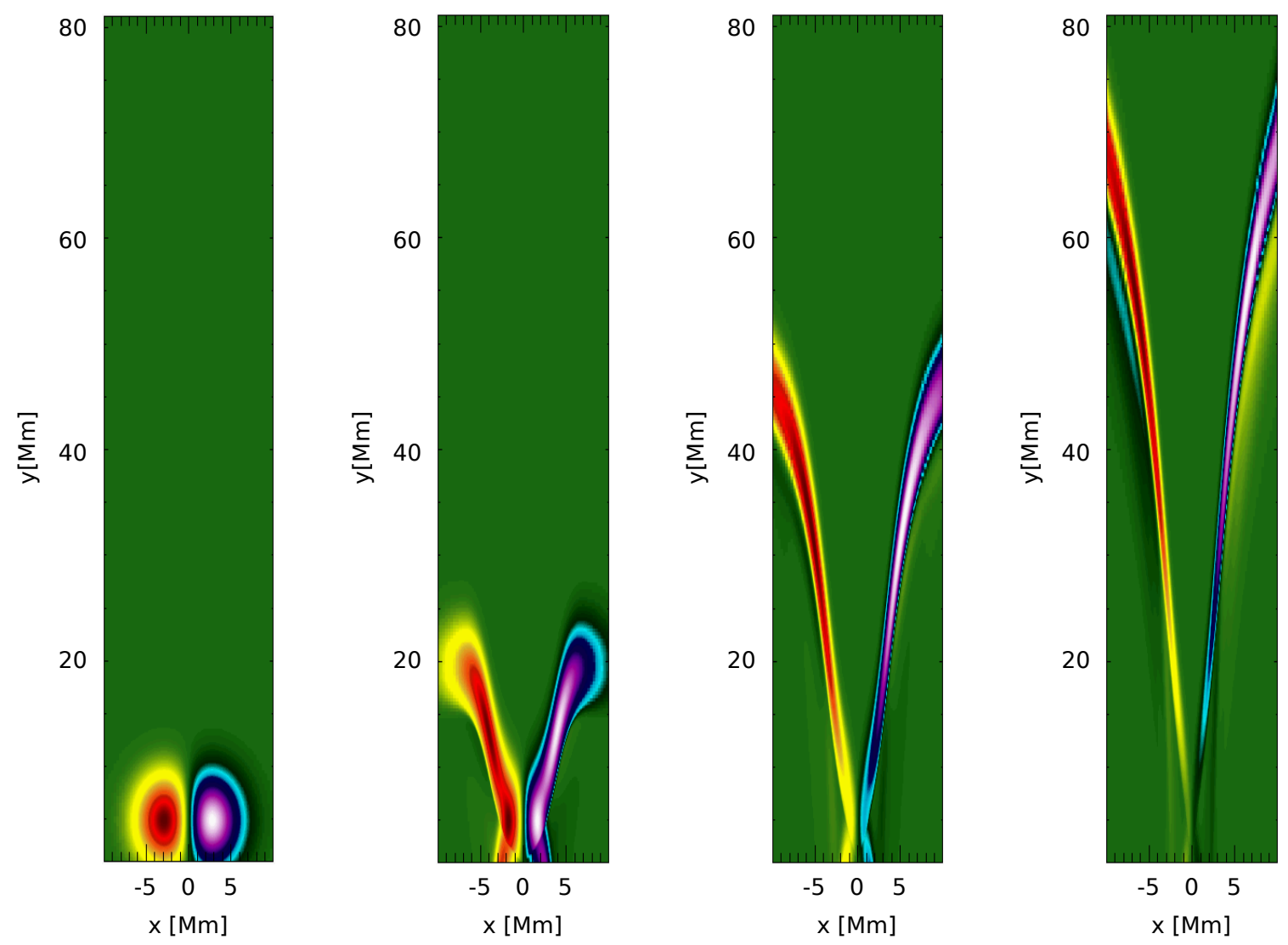

Fig. 5. Time evolution of velocity component $V_{z}$, corresponding to Alfvén waves, at $t=0,10,30$, and $70 \mathrm{~s}$ from left to right.

where $s$ is the coordinate along the magnetic line and the Alfvén speed, $c_{\mathrm{A}}$, is defined as

$c_{\mathrm{A}}(x, y)=\sqrt{\frac{B_{x}^{2}(x, y)+B_{y}^{2}(x, y)}{\mu_{0} \varrho(x, y)}}$.

Here $\varrho(x, y)$ is given by Eq. (16).

\subsection{Numerical solutions}

We solve the 2.5D time-dependent, ideal MHD Eqs. (1)-(4) numerically, making use of the FLASH code (Fryxell et al. 2000; Lee \& Deane 2009; Lee 2013). It is now well tested, fully modular, parallel, multiphysics, open science, simulation code that implements second- and third-order unsplit Godunov solvers with various slope limiters and Riemann solvers as well as adaptive mesh refinement (AMR; see e.g. Chung 2002). The Godunov solver combines the corner transport upwind method for multidimensional integration and the constrained transport algorithm for preserving the divergence-free constraint on the magnetic field (Lee \& Deane 2009). We have used the minmod slope limiter and the Riemann solver (e.g. Murawski 2002; Toro 2006). The main advantage of using AMR technique is to refine a numerical grid at steep spatial profiles while keeping a grid coarse at the places where fine spatial resolution is not essential. In our case, the AMR strategy is based on controlling the numerical errors in a gradient of mass density that leads to reduction of the numerical diffusion within the entire simulation region.

For our numerical simulations, we use a 2D Eulerian box of its height $H=80 \mathrm{Mm}$ and width $W=20 \mathrm{Mm}$. The spatial resolution of the numerical grid is determined with the AMR method. We use the AMR grid with the minimum (maximum) level of the refinement blocks set to 3 (7). The whole simulation region is covered by 12884 blocks. Since every block consists $8 \times 8$ numerical cells, this number of blocks corresponds to 824576 numerical cells.

A spatial grid size has to be less than the typical width of the current sheet along the $x$-direction and the typical wavelength of the Alfvén waves along the $y$-direction, respectively. We find $\min (\Delta x)=\min (\Delta y)=0.04 \mathrm{Mm}$, which satisfies the above-mentioned condition (semi-width of the current sheet is $w_{\mathrm{CS}}=1.0 \mathrm{Mm}$ and the estimated minimal wavelength is approximately $10.0 \mathrm{Mm}$ ). At all boundaries, we fix all plasma quantities to their equilibrium values using fixed-in-time boundary conditions, which lead only to negligibly small numerical reflections of incident wave signals.

\section{Results}

\subsection{Numerical results}

In a current sheet, a velocity perturbation is generated most likely due to reconnection at a height of $5 \mathrm{Mm}$ (cf. Fig. 4), which evolves with height in the stratified solar atmosphere. The initial pulse excites the Alfvén waves. These waves, which are associated with the perturbations in $V_{z}$, propagate along the open magnetic field lines to higher altitudes ( $y$-direction): see the time evolution of $V_{z}$ at $t=0,10,30$, and $70 \mathrm{~s}$ (Fig. 5). The initial pulse (left) spreads into upward and downward propagating waves, which are very well seen at $t=10 \mathrm{~s}$ (second left). Since these waves follow magnetic field lines, which are diverging with height, the left and right profiles of the Alfvén waves move apart with $y$ (see the right most panel). 

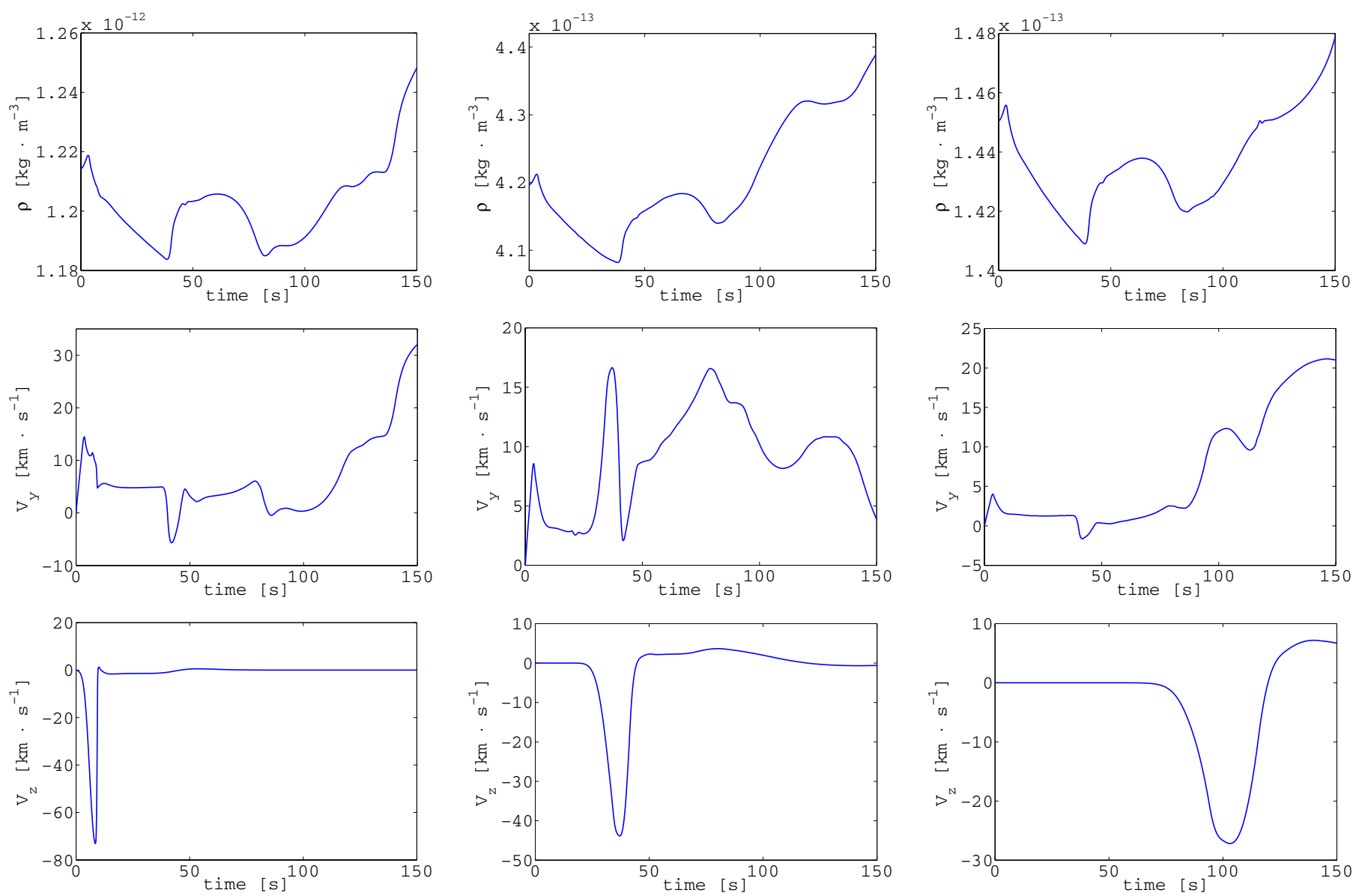

Fig. 6. Top, middle, and bottom panels: spatio-temporal averaged mass density $\varrho$, velocity components $V_{y}$, and $V_{z}$ at three different heights 15,35 , and $55 \mathrm{Mm}$ above the solar surface, respectively.

The associated magnetoacoustic waves produce the mass density perturbations at various heights and also create the vertical plasma dynamics. Some instantaneous mass density, $V_{y}$ and $V_{z}$ velocity variations at 15,35 , and $55 \mathrm{Mm}$ heights along the current sheet above the solar surface are shown in Fig. 6. At each height, we have the temporal signatures of the perturbations of these parameters due to the evolution of waves.

\subsection{Synthetic line-width increment due to Alfvén waves}

In the numerical domain, we have the evolution of Alfvén waves in the Harris current sheet, which propagate along open magnetic field lines in its stratified atmosphere. These Alfvén waves are described by the perturbations in the transverse velocity component $\left(V_{z}\right)$. They result in the magneto-plasma as unresolved nonthermal motions (Banerjee et al. 1998; Chmielewski et al. 2013). The non-thermal motions due to Alfvén waves leave their signature in the line profiles at each height in terms of their broadening. The increment in the line width, therefore, provide the signature of Alfvén wave amplification (Banerjee et al. 1998; O'Shea et al. 2005). As mentioned above, the broadening of line profiles due to Alfvén waves basically lead to the unresolved motions. This contribution to the spectrum, at any particular height in the solar atmosphere, can be due to various wave trains already passed through that detection point. However, these motions are not resolved by the spectrometer though inherent within the spectrum in terms of line broadening. Therefore, we average over the space (entire pulse width) and time (500 s) of various $V_{z}$ signals to know the resultant contribution of the unresolved non-thermal motions. The detailed discussions can be found in Chmielewski et al. (2013). In this subsection, we describe the procedure that we adopt to convert the simulated velocity signals $\left(V_{z}\right)$ into synthetic line width of Fe XII 195.12 А. At any particular height in the model atmosphere, we average the wave velocity amplitude $\left(V_{z}\right)$ over the entire pulse width between $t_{\mathrm{a}}-250$ and $t_{\mathrm{a}}+250 \mathrm{~s}$, where $t_{\mathrm{a}}$ is the arrival time of a wave signal to that particular height (Chmielewski et al. 2013). Therefore, the averaged transverse motions contribute to the non-thermal unresolved motions at a particular height in the model atmosphere as observed in the form of spectral line profile variation due to Alfvén waves. The averaged wave velocity amplitude is scaled in terms of non-thermal speed as $\xi^{2}=0.5 V_{z}^{2}$ taking the polarization and the direction of the propagation of Alfvén waves with respect to the line-of-sight into consideration(Banerjee et al. 1998). It should be noted that this scaling can be applicable for longer exposures. Our spatio-temporal averaging of velocity signal satisfies this requirement. Using the following formula (Mariska 1992):

$\sigma^{2}=\left[4 \ln 2\left(\frac{\lambda}{c}\right)^{2}\left(\frac{2 k_{\mathrm{B}} T}{m_{\mathrm{i}}}+\xi^{2}\right)+\sigma_{\mathrm{I}}^{2}\right]$

we estimate the synthetic line width (FWHM) of Fe XII ( $\lambda=$ $195.12 \AA ; T_{f}=1.2 \times 10^{6} \mathrm{~K}$ ) as a function of height in the model current sheet where Alfvén waves are present. Here, $\sigma_{\mathrm{I}}$ is the instrumental width associated with the Hinode/EIS spectrometer slit. The observed line width is converted into FWHM and subtracted from the instrumental width. While we derive the synthetic line width of Fe XII 195.12 ̊ from the numerical data, 


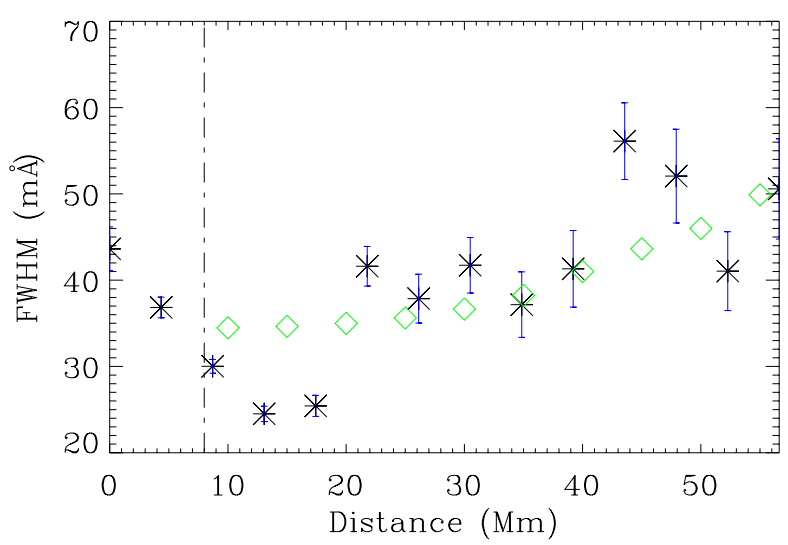

Fig. 7. Variation of synthetic line width with height (diamonds) of Fe XII $195.12 \AA$ as derived by considering the contribution of propagating Alfvén waves in the Harris current sheet, and the observed linewidth broadening along the polar jet (asterisks).

we ignore this factor as it only contains the physical information of thermal and non-thermal motions. It is clear from Fig. 7 (diamonds) that the synthetic line width resulting from the contribution of Alfvén waves grows with height, and its trend matches the variation of the observed line width (cf. Fig. 2, bottom panel; beyond $10 \mathrm{Mm}$ ).

We conclude that the Alfvén waves excited impulsively in our model atmosphere play a similar role like the transverse waves excited within the observed polar jet. Therefore, we precisely constrain the physical mechanisms, i.e. reconnection generated Alfvén waves, which also play a role in triggering the observed jet. The detailed comparison is given in the next subsection. The width of the formed line profile depends both on the thermal and non-thermal contributions in the emitted plasma. It is shown that the heating plays a crucial role in the formation of spectral lines and its equivalent width (e.g. Peter et al. 2006). In the present observational baseline, we have found that our less intense jet does not show much temperature variation along its extended part in the corona as observed by Hinode/EIS. It is only clearly visible in Fe XII $195.12 \AA$ line, while it is not evident in cooler ( $\mathrm{Si}$ VII $275.35 \AA, \log T_{f}=5.0 \mathrm{MK}$ ) as well as hot (Fe XV 284.16 $\left.\AA, \log T_{f}=6.4 \mathrm{MK}\right)$ lines. This means that the heating episode quickly subsides after the reconnection generated origin of the jet. When it is scanned through Hinode/EIS 2 "-slit, it has already reached its maximum height in the polar corona with the plasma maintained around inner coronal temperature of 1.0 MK. Therefore, the thermal width in the formed line profiles of Fe XII 195.12 ̊ (both observed and spectral) can be considered as a constant width equivalent to the formation temperature of Fe XII line, i.e. $T_{f}=1.2 \times 10^{6} \mathrm{~K}$. The nonthermal contribution and resultant line broadening, therefore, result from the Alfvén wave amplification along the jet in its stratified atmosphere.

\subsection{Comparison with the observational data}

The observed jet is triggered by the reconnection between lowlying bipolar loops at its base, and pre-existing open field lines of the polar corona. This forms the typical inverted Y-shape configuration of the jet, and a vertical Harris current sheet along the jet body. The X-type reconnection point can be created somewhere at coronal heights above the inverted Y-shape base of the jet. We model this vertical Harris current sheet as an upper part of the jet where waves and plasma perturbations occur. We assume that the magnetic reconnection generates a velocity pulse, which results in Alfvén waves propagation along the coronal jet.

In the observations, we find the reconnection point at a height 5-10 Mm from the base of the jet where maximum downflow speed is converted into blue shift at higher heights along the jet. Up to this height, the measured FWHM is almost constant. It grows significantly beyond this height, and maximizes up to $50 \mathrm{Mm}$ height along the jet. As noted in Sect. 2, this provides the most likely signature of Alfvén wave growth along the jet body beyond the reconnection point.

In our model, the transverse velocity perturbations are generated due to the reconnection in the lower part of the Harris current sheet, which correspond to Alfvén waves. Although, we only include the reconnection generated velocity pulse in the vertical current sheet of the jet. We obtain the time signatures of Alfvén waves at each height, and convert them into the unresolved non-thermal velocity. Since mass density decreases with height along the open field lines, Alfvén waves are amplified, contributing more to the non-thermal motions at higher altitudes. This results in the line width growth with height of any observed line profile in the stratified solar atmosphere. The synthesized FWHM (line width) due to the contribution of Alfvén waves propagating in the current sheet along the jet body. The synthetic FWHM also shows a similar increasing trend up to $y=55 \mathrm{Mm}$ (cf. Fig. 7). It is constant up to $y=15 \mathrm{Mm}$ above the reconnection point. Beyond the reconnection point, the observed FWHM profile along the polar jet (Fig. 3, right panel) increases. This confirms that the Alfvén waves are impulsively excited within the typical jet morphology due to magnetic reconnection. Alfvén waves propagate along the jet's open magnetic field lines, and further leave their signatures in the form of the observed line width variation. Moreover, the excited slow magnetoaoustic waves perturb the plasma and result in the vertical flows along the magnetic field lines, which cause the jet plasma dynamics. In conclusion, our model supports the scenario of the evolution of non-linear Alfvén waves in the polar coronal jet, and the associated flows that may contribute to its plasma evolution. However, it should be noted that we do not consider the jet's plasma evolution due to heating or direct $\boldsymbol{j} \times \boldsymbol{B}$ force, which may also contribute initially in its formation.

\section{Discussions and conclusion}

Using the Hinode/EIS data, we observe that the magnetic reconnection takes place at the coronal height within a polar jet. Above and below the reconnection point, the plasma shows upflows $\left(-7 \mathrm{~km} \mathrm{~s}^{-1}\right)$ and downflows $\left(+5 \mathrm{~km} \mathrm{~s}^{-1}\right)$, respectively. This confirms the formation of a typical reconnection-generated coronal jet. We also find the line-width increment (Fe XII 195.12 $\AA$ ) along the jet body beyond the reconnection point as a likely signature of the impulsively generated Alfvén waves. The observed line width increment with height matches with its theoretically estimated values, obtained by considering the contribution of Alfvén waves amplitude in the unresolved non-thermal motions of the plasma jet. The inference of this matching provides the physics of Alfvén waves, which are driven within the coronal jets. The reconnection within the vertical Harris current sheet along the jet's body trigger the longitudinal and transverse perturbation, which are associated with fast magnetoacoustic and Alfvén waves. The Alfvén waves leave their signatures collectively in the form of spectral line broadening with height, while the fast magnetoacoustic waves form the plasma flows in the jet. Linear and non-linear Alfvén waves can also be generated at the 
photosphere by granular motions. The region between the photosphere and the transition region acts as an Alfvénic resonator (e.g. Matsumoto \& Shibata 2010). In the present case, we do not find any observational signature of Alfvén waves below $10 \mathrm{Mm}$ within the jet. Therefore, we conjecture that the Alfvén waves are impulsively triggered above the reconnection site lying between 5-10 Mm above the footpoint of the jet. The photospheric Alfvén waves generated by granular motions have typical initial amplitude of a few $\mathrm{km} \mathrm{s}^{-1}$. When they propagate higher into the stratified atmosphere, they do amplify. However, in the present case, the Alfvén waves are triggered in situ at coronal height and possess the larger instantaneous amplitudes $\left(>10 \mathrm{~km} \mathrm{~s}^{-1}\right)$. Therefore, we abbreviate them as impulsively generated nonlinear Alfvén waves propagating along the polar jet.

The underlying reconnection can drive Alfvén waves, which excite the vertical plasma flow. We did not aim to understand the temporal evolution of jet plasma and its heating as previously explored by various models (e.g. Moreno-Insertis et al. 2013). Instead, we aim to understand the signature of Alfvén wave dynamics and associated vertical flows along the jet. We find the clues of both in the form of line-width increment and Doppler velocity distribution (blue shift) along the jet at various heights. For this purpose, we select the Hinode/EIS scanning spectral observations to understand the spatial variation of emission (flux) and plasma properties (flows and width) along the jet. The jet was initially launched due to the reconnection, which may also liberate the heat. The heat flux may be subsided during the whole half-life time of the jet when it has already reached a maximum altitude in the polar corona. The impulsive launch of the jet might leave other physical processes for rest of the time in space along with the jet, i.e. Alfvén waves and associated vertical flows. We observed these two physical processes through EIS spectroscopic scan data, which also confirm the model. Our model is simple in the sense that it does not include the radiative or thermal conductive losses. As a result, this model may not fully describe the reconnection generated heating and related additional plasma evolution within the jet as we do not include the thermodynamical losses in the governing energy equation. In general, the non-consideration of these terms may not affect the properties of Alfvén waves that evolved along the jet. However, they do affect the mass density and temperature evolution of the jet plasma. In our case, the jet is visible in the inner coronal temperature of 1.0 MK, and it is not a very hot evolution of the jet. Moreover, weak EUV emissions also suggest that plasma is not well evolved along the jet spine and it is not a bulky jet as is the case for X-ray jets and surges. To understand and compare the properties of the triggered Alfvén waves, we consider the simplified ideal situation in our model by ignoring the losses. These thermodynamical losses will be considered in our future works in which we will examine their effects on the evolution of temperature and density within the jet.

There is only one report in the imaging X-ray observations from Hinode/XRT, which shows that the small amplitude Alfvén waves propagating from photosphere into the corona can drive the X-ray jets (Cirtain et al. 2007). These important detections are debated by Van Doorsselaere et al. (2008a,b). There are several attempts to model the polar jets in terms of the evolution of Alfvén waves (Kaghashvili 2009; Pariat et al. 2009, 2015; Hollweg \& Kaghashvili 2012, and references cited therein). These models are purely analytical/numerical without any observational support. Nevertheless, they typically reveal the physics of the jet-forming regions as to how these waves can be excited within the typical magnetic field configurations of these jets. Nishizuka et al. (2008) proposed a model of the coronal jets by extending the model of Yokoyama \& Shibata (1995) and found that direct reconnection generated forces can drive both the plasma jet and Alfvén waves at its base. Recently, Kayshap et al. (2013a,b) reported that the reconnection in the lower atmosphere can generate the pulse train and associated slow shocks to drive the surge plasma. Shibata et al. (2007) also suggested three types of the processes for the jet formation caused by the reconnection at different heights. Depending upon the height of magnetic reconnection in the pre-existing magnetic field configuration of the jet, the hot X-ray, EUV, and cool jets as well as $\mathrm{H}_{\alpha}$ surges can be triggered in the coronal, transition region, and chromospheric reconnection, respectively. Therefore, reconnection can be an efficient mechanism to drive the waves/pulses within the confined magneto-plasma system (i.e. various jets), which can also play a role to power them. Alfvén waves are well studied both in theory and observations in driving the smallscale chromospheric jets (e.g. spicules; Kudoh \& Shibata 1999; McIntosh et al. 2011).

In the present work, we have specifically made an effort to find the signatures of reconnction within a jet at a certain height from where the impulsively excited Alfvén waves and plasma motions are evident. We find a good match between the observed scenario and the given physical model of impulsive excitation of Alfvén waves within the vertical Harris current sheet. Our model has the appropriate temperature conditions as well as stratified atmosphere in $2.5 \mathrm{D}$ framework, along with a consideration of the transition region, which plays an important role in reflecting the Alfvén waves. The transition region has an important implication for contribution in non-thermal motions of the corona and in the formation of spectral lines provided they are excited below the transition region. In the present case, the site for impulsive energy release lies in the inner corona. Therefore, the excited Alfvén waves propagating in the outwards direction do not have any influence on the transition region. Moreover, the appropriate temperature conditions also set the stratification within the model atmosphere, which result in the appropriate growth of the Alfvén waves. Therefore, its contribution to the non-thermal motions and corresponding synthetic line broadening are adequately examined and studied. They also match the observed line width variation along the polar jet.

In conclusion, the numerical simulations show that the perturbations in the transversal component of velocity above the reconnection point within the current sheet (the upper part of the jet and its reconnection site) can impulsively excite Alfvén waves. This can also trigger the plasma jet higher in the polar coronal hole as vertical plasma flows associated with magnetoacoustic waves. The inferred contribution of simulated Alfvén waves and their synthetic line widths match the observed nonthermal line broadening along the jet. This provides a direct spectroscopic signature of the reconnection generated Alfvén waves within the polar jet in its typical magnetic field configuration (i.e. the Harris current sheet and its X-type reconnection site).

Acknowledgements. The authors thank the anonymous referee for constructive comments. A.K.S. thanks the visiting scientist travel grant from Prof. K. Murawski, UMCS, Lublin, Poland during September-October 2014 where this work was initiated, and he also acknowledges Shobhna for all her support. We acknowledge the use of Hinode/EIS observations in the present study. This work has granted an access to the HPC resources of CINES under the allocation 2012 D0 046331 \& 2013 D0046331 made by GENCI (Grand Equipement National de Calcul Intensif). P.J. acknowledges support from the Grant P209/12/0103 of the Grant Agency of the Czech Republic. P.J. and K.M. also thank the Marie Curie FP7-PIRSES-GA-2011-295272 Radiophysics of the Sun project. The authors also express their thanks to Piotr Chmielewski for his help with numerical data processing. The FLASH code used in this work was in part developed by the 
DOE-supported ASC/Alliances Center for Astrophysical Thermonuclear Flashes at the University of Chicago.

\section{References}

Banerjee, D., Teriaca, L., Doyle, J. G., \& Wilhelm, K. 1998, A\&A, 339, 208 Bemporad, A., \& Abbo, L. 2012, ApJ, 751, 110

Chmielewski, P., Srivastava, A. K., Murawski, K., \& Musielak, Z. E. 2013, MNRAS, 428, 40

Chmielewski, P., Srivastava, A. K., Murawski, K., \& Musielak, Z. E. 2014, Acta Physica Polonica A, 125, 158

Chung, T. J. 2002, Computational Fluid Dynamics (Cambridge University Press)

Cirtain, J. W., Golub, L., Lundquist, L., et al. 2007, Science, 318, 1580

Culhane, J. L., Doschek, G. A., Watanabe, T., et al. 2006, Proc. SPIE, 6266, 62660

De Pontieu, B., McIntosh, S. W., Carlsson, M., et al. 2007, Science, 318, 1574

Dwivedi, B. N., \& Srivastava, A. K. 2006, Sol. Phys., 237, 143

Dwivedi, B. N., Srivastava, A. K., \& Mohan, A. 2014, PASJ, 66, S13

Filippov, B., Golub, L., \& Koutchmy, S. 2009, Sol. Phys., 254, 259

Fryxell, B., Olson, K., Ricker, P., et al. 2000, ApJS, 131, 273

Galsgaard, K., \& Roussev, I. 2002, A\&A, 383, 685

Goossens, M., Terradas, J., Andries, J., Arregui, I., \& Ballester, J. L. 2009, A\&A, 503,213

Goossens, M., Andries, J., Soler, R., et al. 2012, ApJ, 753, 111

Harrison, R. A., Hood, A. W., \& Pike, C. D. 2002, A\&A, 392, 319

Hollweg, J. V., \& Kaghashvili, E. K. 2012, ApJ, 744, 114

Jess, D. B., Mathioudakis, M., Erdélyi, R., et al. 2009, Science, 323, 1582

Jelínek, P., Karlický, M., \& Murawski, K. 2012, A\&A, 546, A49

Kaghashvili, E. K., Quinn, R. A., \& Hollweg, J. V. 2009, ApJ, 703, 1318

Kayshap, P., Srivastava, A. K., Murawski, K., \& Tripathi, D. 2013a, ApJ, 770, L3

Kayshap, P., Srivastava, A. K., \& Murawski, K. 2013b, ApJ, 763, 24

Kudoh, T., \& Shibata, K. 1999, ApJ, 514, 493

Lee, D. 2013, J. Comput. Phys., 243, 269

Lee, D., \& Deane, A. E. 2009, J. Comput. Phys., 228, 952

Mariska, J. T. 1992, Cambridge Astrophysics Series (New York: Cambridge University Press)

Mathioudakis, M., Jess, D. B., \& Erdélyi, R. 2013, Space Sci. Rev., 175, 1
Matsumoto, T., \& Shibata, K. 2010, ApJ, 710, 1857

McIntosh, S. W., de Pontieu, B., Carlsson, M., et al. 2011, Nature, 475, 477

Moreno-Insertis, F., \& Galsgaard, K. 2013, ApJ, 771, 20

Murawski, K. 2002, Analytical and Numerical Methods for Wave Propagation in Fluid Media (Singapore: World Scientific)

Murawski, K., Solov'ev, A., Musielak, Z. E., Srivastava, A. K., \& Kraśkiewicz, J. 2015a, A\&A, 577, A126

Murawski, K., Srivastava, A. K., Musielak, Z. E., \& Dwivedi, B. N. 2015b, ArXiv e-prints [arXiv: 1505.03793]

Nakariakov, V. M., Arber, T. D., Ault, C. E., et al. 2004, MNRAS, 349, 705

Nakariakov, V. M., Pascoe, D. J., \& Arber, T. D. 2005, Space Sci. Rev., 121, 115

Nishizuka, N., Shimizu, M., Nakamura, T., et al. 2008, ApJ, 683, L83

Nisticò, G., \& Zimbardo, G. 2012, Adv. Sp. Res., 49, 408

Nisticò, G., Bothmer, V., Patsourakos, S., \& Zimbardo, G. 2009, Sol. Phys., 259, 87

Okamoto, T. J., Tsuneta, S., Berger, T. E., et al. 2007, Science, 318, 1577

O'Shea, E., Banerjee, D., \& Doyle, J. G. 2005, A\&A, 436, L35

Pariat, E., Antiochos, S. K., \& DeVore, C. R. 2009, ApJ, 691, 61

Pariat, E., Dalmasse, K., DeVore, C. R., Antiochos, S. K., \& Karpen, J. T. 2015, A\&A, 573, A130

Pascoe, D. J., Wright, A. N., \& De Moortel, I. 2010, ApJ, 711, 990

Pascoe, D. J., Wright, A. N., \& De Moortel, I. 2011, ApJ, 731, 73

Peter, H., \& Judge, P. G. 1999, ApJ, 522, 1148

Peter, H., Gudiksen, B. V., \& Nordlund, A. 2006, ApJ, 638, 1086

Priest, E. R. 1982, Solar Magnetohydrodynamics (London, England: D. Reidel Publishing Company)

Shibata, K. 1982, Sol. Phys., 81, 9

Shibata, K., Nakamura, T., Matsumoto, T., et al. 2007, Science, 318, 1591

Somov, B. V. 1994, Space Sci. Rev., 70, 161

Srivastava, A. K., \& Murawski, K. 2011, A\&A, 534, A62

Srivastava, A. K., \& Goossens, M. 2013, ApJ, 777, 17

Tomczyk, S., McIntosh, S. W., Keil, S. L., et al. 2007, Science, 317, 1192

Toro, E. F. 2006, Int. J. Num. Meth. Fluids, 52, 433

Van Doorsselaere, T., Brady, C. S., Verwichte, E., \& Nakariakov, V. M. 2008a, A\&A, 491, L9

Van Doorsselaere, T., Nakariakov, V. M., \& Verwichte, E. 2008b, ApJ, 676, L73

Vernazza, J. E., Avrett, E. H., \& Loeser, R. 1981, ApJ, 45, 635

Yokoyama, T., \& Shibata, K. 1995, Nature, 375, 42

Young, P. R., Watanabe, T., Hara, H., \& Mariska, J. T. 2009, A\&A, 495, 587 